\title{
Aplicación de indicadores de conocimiento sobre biodiversidad para el diagnostico y comparación de colecciones biológicas.
}

\author{
Martha Y. Vallejo' y Alberto Acosta $\mathrm{PhD}^{2}$ \\ ${ }^{1,2}$ Pontificia Universidad Javeriana, Facultad de Ciencias, Departamento de Biología. Unidad de Ecología y Sistemática (UNESIS). \\ Correspondencia:1vallejom@javeriana.edu.co ${ }^{2}$ laacosta@javeriana.edu.co \\ Recibido: 27-04-2005 / Aceptado: 05-10-2005
}

\begin{abstract}
Resumen
La mayoría de colecciones biológicas de los museos en Colombia carecen de elementos de diagnóstico, lo que impide analizarlas y determinar su estado en términos cuantitativos y cualitativos. Para suplir esta carencia se proponen indicadores de biodiversidad aplicables a las bases de datos de las colecciones biológicas que permitan a los museos definir sus condiciones, prioridades y registrar su aporte a la biodiversidad. Es así, como existen criterios básicos para elaborar dichos indicadores: validez científica, disponibilidad y fiabilidad de los datos, este último incluye; representatividad, sensibilidad a cambios, sencillez, comparabilidad, relevancia y utilidad entre otros. Estos criterios son necesarios para soportar cada indicador como parte de la hoja metodológica.

Los indicadores de conocimiento sobre biodiversidad propuestos sirven para comparar el mismo grupo biológico: (i) entre diferentes colecciones, como el caso de estudio escleractinios del Museo Javeriano - MUJ y Museo de Historia Natural Marina de Colombia- MHNMC; (ii) comparar una colección frente a un total reportado para el país o el mundo. Los indicadores usados fueron los de: representatividad y complementariedad, tanto taxonómica como geográfica, especies en peligro, identificación taxonómica, completitud de datos, intensidad de muestreo e índice de metadatos.

Los indicadores utilizados arrojaron los siguientes resultados para la colección del MUJ: especies con las que cuenta, 30 corales hermatípicos; carece de, 52 corales ahermatípicos en muestreos en 3 ecorregiones del país; distribución temporal de colecta, 91.6\% de las colectas recientes; distribución espacial de colecta, 6 ecorregiones a lo largo del Caribe colombiano; representatividad taxonómica 50\% frente al MHNMC y $26 \%$ frente al total reportado en el Caribe; nivel de curatoría, $94.4 \%$ de ejemplares identificados hasta especie; especies en amenaza presentes, 17.6\%; familia con mas repeticiones, Faviidae y mas representativa, Agariciidae. El uso de indicadores incrementa la utilización y divulgación de la biodiversidad de un país y permite la especialización de la colección a través de la información documentada, permitiendo a los museos generar un diagnostico por colección y establecer prioridades de manejo y optimización de los recursos económicos.
\end{abstract}

Palabras Claves: Caribe colombiano, colecciones biológicas, diagnóstico, escleractínios, indicadores de biodiversidad, museos. 


\begin{abstract}
Most biological collections from Colombian museums lack diagnosis, which prevent to anmize and to know it real state in qualitative and quantitative terms. To solve this problem it has been proposed some biodiversity indicators, which can be used on biological collections data bases, allowing museums to define it condition, priorities and to register it contribution to country biodiversity.

There are basic criteria to elaborate indicators such as: scientific validation, data availability and reliability; the later including representativity, sensibility, simplicity, relevance and utility among others. They are necessary to support each indicator as part of the methodological sheet.

The indicators proposed are used to compare the same biological group: (i). Between different collections; as this study case: Museo Javeriano - MUJ against Museo de Historia Natural Marina de Colombia- MHNMC and (ii). One Museum against a total reported for the country or the world.

The indicators used were: representativity and complementarity, both in taxonomic as in geographic terms; danger species; taxonomy identification; data completeness, sampled intensity, and metadata index. The indicators showed the following results for the MUJ collection: registred species 30 hermatipic corals; absent species, 52 ahermatipic corals; sampling in three ecoregions in the country, temporal, $91.6 \%$ of the material collected recently, 2004; spatial distribution of the specimens collected, 6 ecoregions along the colombian Caribbean; taxonomic representativeness, 50\% against MHNMC and 26\% against a total documented in the Caribbean; curatory level, $94.4 \%$ material identify to species; danger species included in the collection, $17.6 \%$; family with more repetitions, Faviidae, and most representative; Agariciidae. The use of indicators increases the collection utility, disclosure (country biodiversity) and specialization through the documented information. They allows museums to make diagnosis for each collection in order to establish management priorities and optimization of the economical resources.

Key Words: biodiversity indicators, diagnostic, biologic collection, museum, scleractinias, colombian Caribbean.
\end{abstract}

\title{
Introducción
}

El diseño original de la hoja metodológica, criterios y condiciones para elaborar un índice indicador de conocimiento sobre biodiversidad; fue propuesto por la Unidad de Política Ambiental del Departamento Nacional de Planeación en 1998 para el uso de un sistema de indicadores de gestión y planificación ambiental (SIPSA), "Indicadores Ambientales y de Sustentabilidad para América Latina y el Caribe" (1).

En el 2001 se implementaron, para ser utilizados en Colombia. Los puntos a considerar en la elaboración de la hoja metodológica, de acuerdo con Ortiz et al. (2); fueron: 1. Definición; 2. Pertinencia; 3. Unidad de medida; 4. Fórmula; 5. Descripción metodológica; 5.1. Proceso de cálculo; 5.2. Limitaciones; 6. Cobertura; 7. Escala; 8. Relación con otros indicadores; 9. Fuente

de los datos; 10. Disponibilidad de los datos; 10.1. Existencia de series históricas; 10.2. Nivel de actualización de los datos; 10.3. Estado actual de los datos; 10.4 . Forma de presentación de los datos; 11. Periodicidad de los datos;12. Posibles entidades responsables del indicador; 13. Documentación relacionada; 14. Ejemplo numérico y 15. Ejemplo gráfico.

Los indicadores se apoyan en los datos de las colecciones biológicas, ya que estas, al ser bancos de información que dependen de las condiciones de los ejemplares colectados, de la amplitud y la conservación de los mismos; y de la fiabilidad de sus datos, son las que representan el patrimonio en términos de biodiversidad de un país (3). 
En los museos ha sido difícil realizar avances significativos alrededor del manejo de la información de las colecciones, porque no se han invertido recursos económicos y tecnológicos en su desarrollo; capacitación, implementación de bibliografía, líneas de investigación con ejemplares, etc, que admitan la generación, dirección y uso de la información en general, y a su vez no existe un diagnostico de su estado.

El desarrollo de indicadores de biodiversidad permite conocer el estado de la colección de un museo, al igual que el seguimiento de logros en la generación de análisis y uso de la información documentada en las bases de datos de las colecciones, articulándola, mejorándola e integrándola para complementar los inventarios de especies en el país (4). Estos indicadores basados en índices, al ser expresiones cuantitativas y cualitativas de información, se constituyen en apoyos metodológicos para realizar un diagnóstico $\mathrm{y}$ avances en una colección de manera tal que sus logros puedan ser monitoreados por la institución en un periodo de tiempo (4).

Los indicadores de biodiversidad son, en términos generales, instrumentos que orientan y alimentan la reflexión sobre los patrones y procesos en la generación del conocimiento científico (5). Cuando se evalúan, comparan e integran datos de diferentes museos, es indispensable que las colecciones involucradas sean afines en cuanto a su área de influencia, actividades de colecta e intereses taxonómicos, para que los datos compartidos presenten perfiles similares y tengan sentido (6). La eficacia de usar dichos indicadores de biodiversidad, es debido a que estos ofrecen un apoyo para realizar un diagnóstico, si se combinan entre sí (5).

Los indicadores sirven para (i) especializar una colección biológica, (ii) planear investigaciones futuras, (iii) conocer la relevancia de cada una de las colecciones; definir el cubrimiento en términos taxonómicos y geográficos, (iv) cuantificar y cualificar los datos tomados en campo, (v) ver las carencias de la colección biológica en estudio, (vi) conocer la información de las colecciones biológicas; divulgar, recopilar datos en una colección biológica para un fin especifico, (vii) obtener representatividad taxonómica y geográfica frente al total en el país, (viii) observar el esfuerzo de colecta en las colecciones biológicas evaluadas, (ix) integrar la información y contribuir en los procesos de biodiversidad, (x) facilitar decisiones en cuanto a biodiversidad en nuestro país, teniendo en cuenta por ejemplo especies en vía de extinción (7).

De esta manera, la evaluación de información sobre organismos colectados en el territorio colombiano es importante para tomar decisiones relacionadas con biodiversidad, conservación, para diseñar estrategias de colecta, reguladas entre instituciones, para completar el inventario nacional, y contar con datos fiables para otros estudios a diferentes escalas espacio-temporales (6).

Existen varios indicadores de biodiversidad, los cuales pueden ser aplicados en diferentes contextos. En Europa los indicadores han sido utilizados para evaluar la calidad del medio ambiente; ejemplo de ello son entidades como la Comisión Económica para $\mathrm{Eu}-$ ropa de las Naciones Unidas CEPE, que aplica 150 indicadores agrupados en 19 áreas y subáreas de interés ambiental, cada una constituida por uno o más indicadores (8). Las áreas con indicadores aplicados son: naturaleza, recursos, actividades humanas que afectan al medio, calidad del medio, especies, hábitats, gestión ambiental, cambio climático, disminución de la capa de ozono, acidificación, ozono troposférico, calidad del aire urbano, gestión de residuos, calidad de aguas continentales, litoral, aguas marinas, naturaleza y biodiversidad (8).

El objetivo de esta publicación es demostrar la utilidad de los indicadores de conocimiento sobre biodiversidad, aplicados a las bases de datos de las colecciones biológicas y el diagnóstico que se infiere a través de ellos para trazar objetivos y políticas específicas en los museos. 


\section{Materiales y métodos}

Para abordar el presente estudio se uso la información de 130 registros biológicos de Escleractinios de la colección de referencia del Museo Javeriano de Historia Natural Lorenzo Uribe S.J. (MUJ) y se comparó a dos niveles: (i) contra los 284 registros biológicos del mismo grupo biológico referenciados en la colección del Museo de Historia Natural Marina de Colombia (MHNMC), y (ii) contra la lista de 115 especies de Escleractinios reportados para el Caribe colombiano (9). Se establecieron parámetros de comparación a partir de las bases de datos de las colecciones biológicas; MUJ-MHNMC y se manejaron variables tales como: integridad taxonómica, cobertura geográfica, extensión temporal, acrónimo, código de la institución, numero del ejemplar y que las colecciones fuesen del mismo grupo biológico Escleractinios.

Se aplicaron las fórmulas de tres tipos de indicadores de biodiversidad a los datos de los registros: cantidad, calidad e intercambio de información de colecciones biológicas (Tabla 1).

Los resultados obtenidos de algunos indicadores oscilan entre cero y la unidad (complementariedad), y los demás se interpretaron porcentualmente bajo la siguiente escala: 0 a 35 bajo, de 36 a 70 medio y de 71 a 100 alto.

\section{Resultados}

La colecta de los ejemplares del orden Escleractinios (MUJ) fue realizada entre 1999 y el 2004. Pese a ser en un lapso corto, se observa colecta interrum-pida. Se presentó mayor intensidad de colecta y por ende mayor número de especies en el 2004, época donde se evidenció de igual forma más representa-tividad taxonómica. Se identificaron 11 familias, 19 géneros y 30 especies de Escleractinios para el MUJ. Existe ausencia de corales ahermatipicos en la colección del MUJ, mientras que el MHNMC referenció 27 especies frente a 52 reportados para el Caribe colombiano (Figura 1A).
No obstante el MUJ tiene exclusividad de 23 juveniles hermatípicos y 7 especies únicas referenciadas para el país. Se obtuvó una representación taxonómica alta al comparar los taxones de Escleractinios del MUJ frente al MHNMC y baja contra el total reportado para el Caribe colombiano (Figuras 1 A y B).

La tendencia para los dos casos fue: a mayor jerarquía, es decir a medida que incrementaba la clasificación en términos de familia, género y especie, menor representatividad taxonómica. Las especies referenciadas en el MUJ fue del 50\% contra las existentes en el MHNMC y $26 \%$ del total reportado para el Caribe colombiano (Figuras $2 \mathrm{~A}$ y B).

E1 90\% de los ejemplares del MUJ se han colectado en tres ecorregiones del Caribe colombiano, San Andrés, Magdalena y Tayrona, faltando muestreo en Darién, Guajira y Morrosquillo. Las colecciones de Escleractinios del MUJ y MHNMC a nivel taxonómico, son altamente complementarias. Seis familias, $35.3 \%$, presentaron valores iguales a 1 en complementariedad, evidenciando la exclusividad de estos taxones para el MHNMC y ausencia de los mismos para el MUJ. 23.5\% de los taxones mostraron valores cercanos a 1 . El 29.4\% de las especies con valores cercanos a 0 y el $11.8 \%$ de las familias con valores iguales a 0 , representando para estas familias baja complementariedad. Se evidenció que la colección biológica de escleractinios del MUJ comparte un alto porcentaje de ecorregiones muestreadas con respecto a las ecorregiones en estudio y con las muestreadas por el MHNMC, y por lo tanto la tendencia de complementariedad es considerada baja entre las dos colecciones. La curatoria, entendida como la gestión del taxónomo en este caso es alta (94.4\%). Las especies amenazadas preservadas dentro de la colección fue de $17.6 \%$, siguiendo a UICN. El indicador de completitud en los conjuntos de datos (MUJ y MHNMC) presentó un alto porcentaje de información documentada y almacenada en campos críticos (76.6\%).

www.unicolmayor.edu.co 
Tabla 1. Indicadores de biodiversidad aplicados a la colección del MUJ, MHNMC y spp reportadas para el Caribe colombiano. Los valores varían entre o (ausencia) y 1 (presencia). Spp: Especies. ${ }^{*}$ Aplicado a nivel de familia.

\begin{tabular}{|c|c|c|}
\hline $\begin{array}{l}\text { Indicadores de } \\
\text { Biodiversidad (Índices) }\end{array}$ & Fórmulas & Variables \\
\hline Representatividad taxonómica & $\mathrm{RT}_{i}=$ ISCilSCelx 100 & $\begin{array}{l}\mathrm{SC}_{\mathrm{i}} \text { : \# spp del taxón } \\
\mathrm{SC}_{\mathrm{e}} \text { : \# } \mathrm{Spp} \text {. para Colombia. }\end{array}$ \\
\hline Representatividad geográfica & $R G_{i h}=|A G i h / A G h| \times 100$ & $\begin{array}{l}\text { AGih: \# de ecorregiones } \\
\text { AGh: \# de ecorregiones Caribe colombiano }\end{array}$ \\
\hline${ }^{*}$ Complementariedad taxonómica & $\begin{array}{l}C_{i j t}=I U i j c / C i j c \mid \times 100 \\
U_{i j t}=S_{i c} \times S_{j i c}-2 V_{i j c}\end{array}$ & $\begin{array}{l}\text { Uijc : \# de spp reportada en solo } 1 \text { de las colecciones } \\
\text { Cijc: \# de spp en solo } 1 \text { de las colecciones } \\
\text { Sis: \# de sppj } \\
\text { Sic: \# de spp i } \\
V_{\text {je: }} \text { \# de spp simultaneas en las dos colecciones }\end{array}$ \\
\hline${ }^{*}$ Complementariedad geográfica & $\begin{array}{l}C_{i p h}==\mid U_{i j c / C i j c l} \times 100 \\
\text { Uijh }=S_{i h} \times S_{i h}-2 V_{i j h}\end{array}$ & 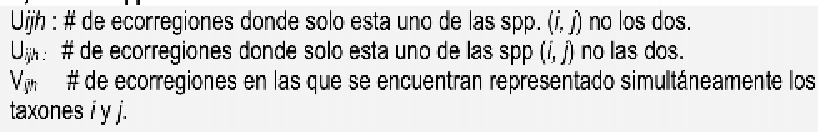 \\
\hline Representatividad especies en peligro & $\mathrm{RAC}=|N E c / N T A| \times 100$ & $\begin{array}{l}\text { Nec: \# spp en peligro representada en la colección } \\
\text { NTA \# total de spp reportada en amenaza por el UICN }\end{array}$ \\
\hline Identificación Taxonómica & & $\begin{array}{l}\text { ITid \# de spp identificados en la colección } \\
\text { RBt \# total de registros biológicos de la colección. }\end{array}$ \\
\hline completitud de datos & $C_{c}=I C d / C t \mid \times 100$ & $\begin{array}{l}\text { Cd: \# de campos críticos llenos en la colección del MUJ } \\
\mathrm{Ct}: \text { es el total de campos críticos. }\end{array}$ \\
\hline $\begin{array}{l}\text { Intensidad de muestreo para registros } \\
\text { biológicos }\end{array}$ & $\mathrm{IMCr}=\mathbf{I N R} t / \mathrm{NL} t \mathrm{IX} 100$ & $\begin{array}{l}\text { NRt : \# total de registros biológicos para cada taxón } \\
\text { NLt : \# total de ecorregiones para cada taxón. }\end{array}$ \\
\hline Intensidad de muestreo para especies & IMtt $=\mathrm{INTt} / \mathrm{NL} f \mathrm{IX} 100$ & $\begin{array}{l}\text { NTt : \# total de spp en cada familia } \\
\text { NLt: \# total de localidades para cada taxón. }\end{array}$ \\
\hline Indicador de metadatos & $\mathrm{NTt}=\mathrm{IMo} / \mathrm{Mt} \mathrm{I} \times 100$ & $\begin{array}{l}\text { Mo: \# de campos obligatorios documentados en la colección } \\
\text { Mt \# total de campos obligatorios en el estándar utilizado. }\end{array}$ \\
\hline
\end{tabular}

Fuente: Inst. Alexander von Humboldt.
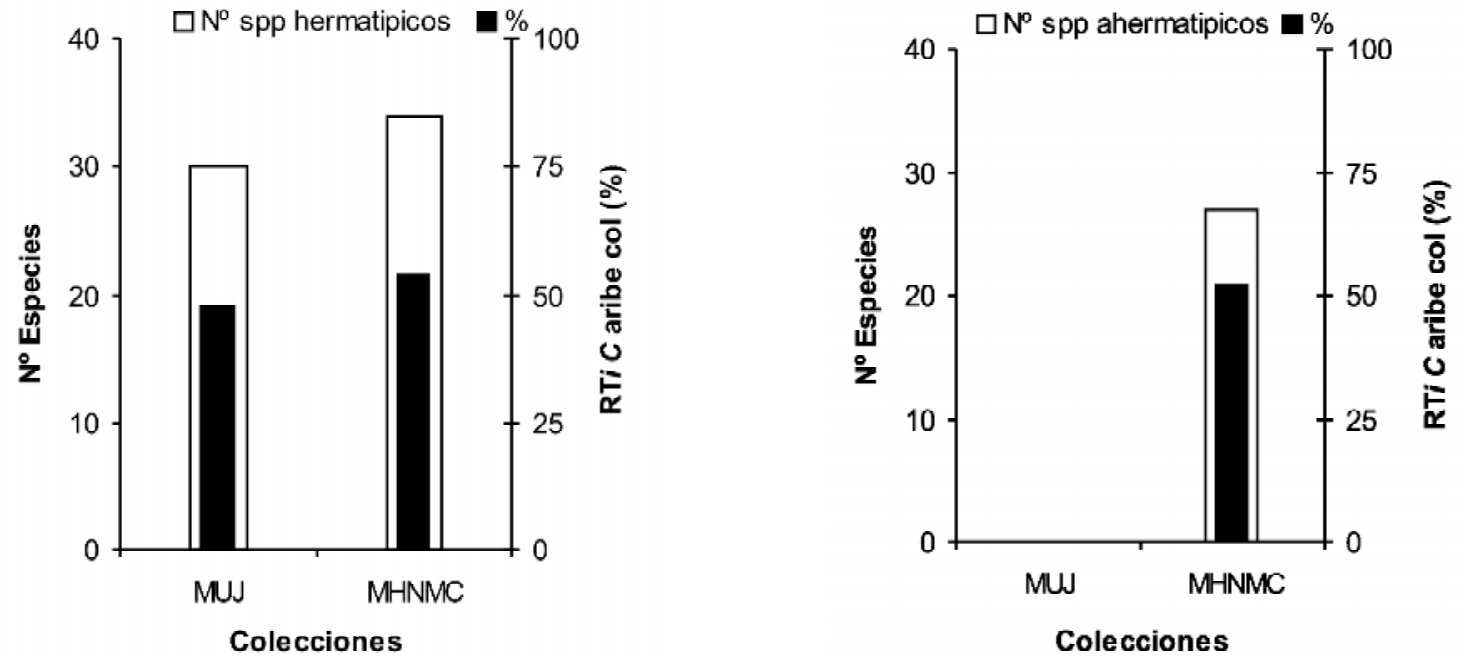

Figura 1. A. Comparación de la representatividad taxonómica de corales ahermatípicos referenciados en el MUJ contra el total de corales ahermatipicos referenciados en la colección del MHNMC. El número de especies de cada colección se presentó en blanco, y el porcentaje de representatividad en negro para las dos colecciones. Rti: Representatividad Taxonómica; F: Familia; G: Género; E: Especie. B. Comparación de la representatividad taxonómica de corales hermatipicos referenciados en el MUJ contra el total de corales hermatípicos referenciados en la colección del MHNMC.

Las Familias con mayor intensidad de muestreo fueron: Faviidae, Poritidae y Mussidae correspondiendo a un $65.3 \%$ del total de registros biológicos (130). Las familias con mayor número de especies

fueron: Faviidae; 10 especies, Mussidae; 4 especies y Agariicidae; 3 especies, aportando el 56.6\% del total de las especies (30). El MUJ cuenta con el $75 \%$ de los campos obligatorios documentados para 


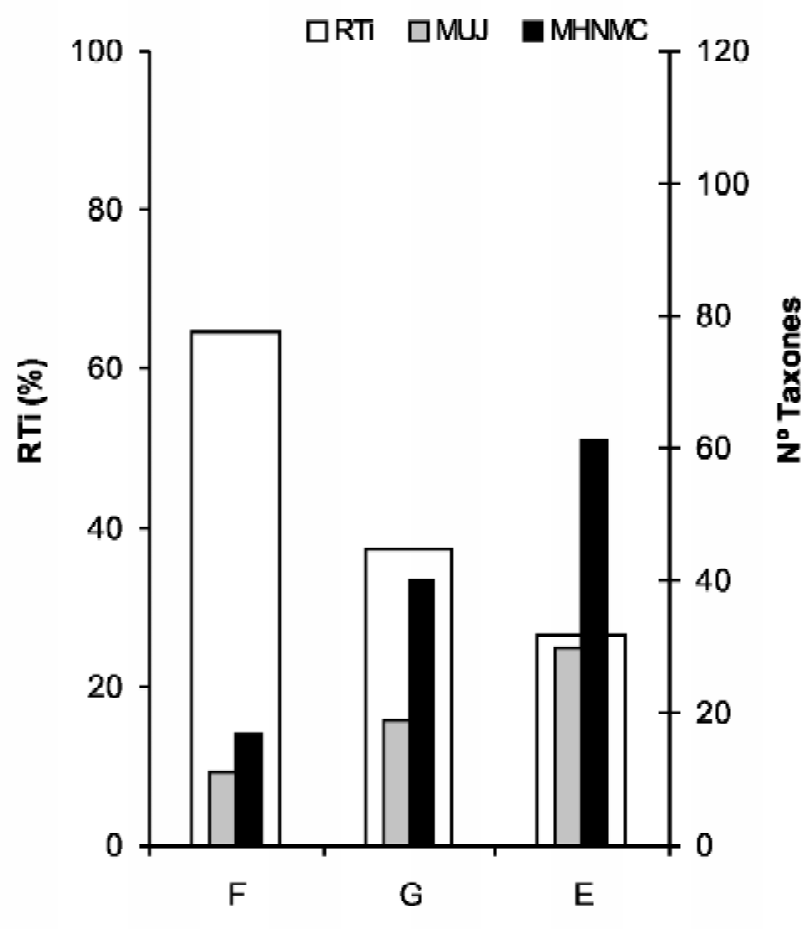

Jerarquia Taxonómica

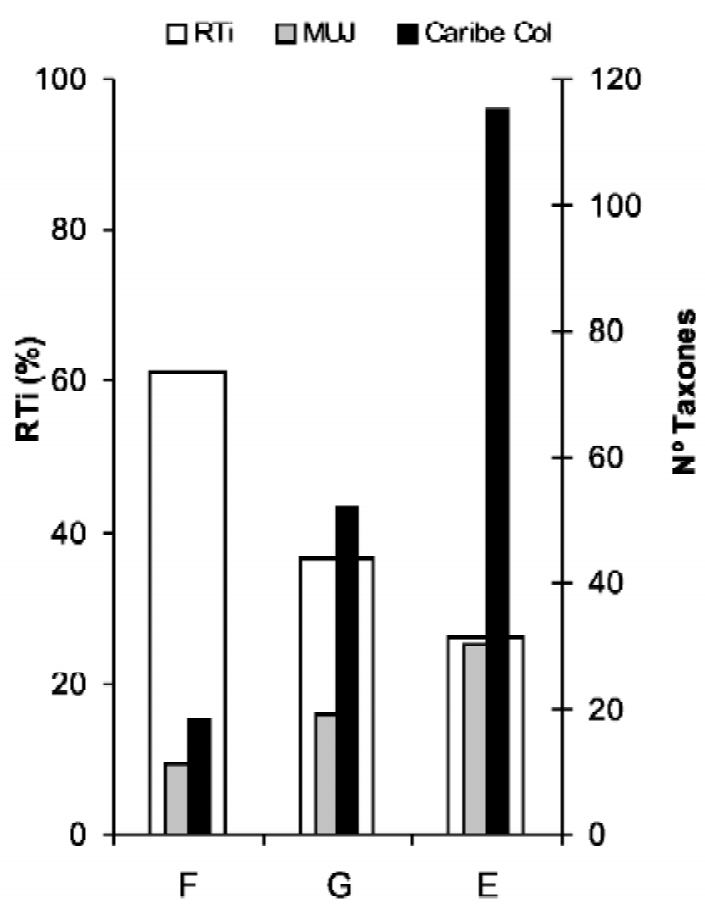

Jerarquia Taxonómica

Figura 2. A. Porcentaje de representatividad taxonómica de los Escleractinios de la colección biológica del MUJ al ser comparado con el MHNMC. B. Porcentaje de representatividad taxonómica de los Scleractinios de la colección biológica del MUJ al ser comparado con el total para el Caribe colombiano. Familia: F, Género: G y Especie: E. El número de taxones de cada colección se encuentra en la parte interna de las barras para las dos colecciones; en gris para el MUJ, y en negro para el MHNMC. El Rti, se presenta en blanco. Rti: Representatividad Taxonómica.

la construcción del metadato, contra un $96 \%$ del MHNMC, al seguir el mismo estándar propuesto para la documentación del conjunto de datos (10).

\section{Discusión}

La selección de indicadores para el diagnóstico de la colección de corales del MUJ, se basó como afirma Hyman et al. (11), en los objetivos que se pretenden en un estudio. Estos son representatividad e inventario taxonómico y la calidad y completitud de la información en las bases de datos (10). El indicador de representatividad taxonómica, mostró valores porcentuales de los taxones almacenados en la colección del MUJ (4), respecto de un total de riqueza estimada para el país y frente a la colección del MHNMC, para el mismo taxón. Los bajos porcentajes de representatividad taxonómica de la colección del MUJ contra el total para Colombia y el MHNMC, se expli- can por la ausencia de corales ahermatípicos, (Figura $1 \mathrm{~A})$, y alto porcentaje relativo ( $90 \%$ ) de registros biológicos de corales hermatípicos, y de fácil colecta en aguas someras (Figura 1B). Además, son un grupo conspicuo en los arrecifes por su tamaño y cobertura a diferencia de los corales ahermatipicos (12).

No obstante la importancia de la colección de escleractinios del MUJ para el país, radica en la exclusividad de sus ejemplares, que son en su totalidad corales hermatípicos juveniles. Sin embargo, el análisis de éste indicador es de cuidado, debido a la fluctuación taxonómica que representa cualquier valor estimado de riqueza (4) y frente a la discrepancia de criterios entre autores en cuanto al número de especies de un taxón dado (6), que en este caso fueron los corales de los géneros: Agaricia, Cladocora, Colpophyllia, Dichocoenia, Diploria, Eusmilia, Favia, Leptoseris, Isophyllastrea, Madracis, 
Manicina, Meandrina, Montastrea, Mussa, Mycetophyllia, Porites, Scolymia, Siderastrea y Tubastrea del Caribe colombiano.

La colecta de los corales se realizó en tres ecorregiones de siete del Caribe colombiano, analizándose que en Colombia en los últimos años, hay sesgo al realizar los muestreos sobre biodiversidad, ejemplo de ello se ve en grupos de organismos, como aves, Renjifo et al. (13), Delgado (14); plantas; IAvH et al. (15), Vivas (6), y avispas parasitoides, Campos (16) y Vivas et al (17). Como lo afirman Soberón et al. (18), las áreas donde más se muestrea están asociadas a algún interés científico, con la influencia de la actividad de institutos de investigación o universidades, con la presencia de vías de comunicación y logística, seguridad, costos y con proyectos estatales de recolección de información.

Por otro lado, las ecorregiones no muestreadas, se relacionan posiblemente como lo afirma Vivas (17), en los recortes de presupuesto para actividades de investigación y en las demoras para los permisos ante los Ministerios en el momento de colectar. Pese, a que se ha muestreado en conjunto con el MHNMC todas las ecorregiones del Caribe colombiano, no se obtuvo distribución geográfica biomaps de los ejemplares colectados, debido a la falta de coordenadas geográficas asociadas a los registros, para con ellos ser interpretada en conjunto con el indicador de complementariedad geográfica de manera confiable (19).

Actualmente existe una seria preocupación en los círculos científicos, acerca de la conservación de la biota, flora, fauna y ambiente, ante la persistente degradación y desaparición de numerosas áreas naturales y especies nativas a nivel mundial (20). Es por eso que cobra importancia la interpretación del indicador de especies amenazadas, que se relaciona con el conocimiento de la biota en peligro para Colombia, y éste dato es de vital importancia, como lo afirma Fernández (21), para la reconstrucción de geanologías, y por su aporte de datos genéticos gracias a las modernas técnicas de genética molecular y a la conservación de las especies en peli- gro. Así mismo, los valores de los indicadores de cantidad de información, dependieron básicamente de la calidad de ésta en las colecciones biológicas, del proceso de integración de los datos, de la cantidad y condiciones de los ejemplares recolectados, de los métodos usados para el ingreso de los datos a la base de datos (22) y de los objetivos específicos que se consideraron en el momento de realizar la curatoría de la colección de corales, tales como: 1. Metas y prioridades propuestas; 2. Planeación estratégica asociada y 3 . Liderazgo (21). La alta proporción de complementariedad de las colecciones a nivel taxonómico con la cuál se obtiene la representatividad total de taxones de corales en los conjuntos de datos evaluados (MUJ y MHNMC), se ve apoyado con lo expuesto por Vivas (17), donde la aplicación del índice de complementariedad taxonómica y geográfica a un conjunto de datos, arroja resultados que demuestran que las colecciones con especies únicas (MUJ) son importantes para la totalidad del inventario taxonómico del país, independiente del número de registros que posea (6). Los resultados de los índices de intensidad de muestreo; especies/ localidad/ MUJ, revelan como lo ha planteado Williams y Gaston (23), que a mayor número de localidades muestreadas y registros, se espera que se encuentre un mayor número de taxones identificados. Es decir, los taxones; Faviidae, Poritidae, Mussidae, Agariicidae y las localidades muestreadas; San Andrés, Magdalena y Tayrona estan directamente relacionados, si se interpretan como la medida del esfuerzo invertido por un investigador en la colecta de entidades biológicas; concepto que corresponde al enfoque usado por investigadores como Colwell y Coddington (24), Hijmans et.al; (25), y Soberón et. al; (18). Sin embargo, los valores arrojados, no deben confundirse con las múltiples repeticiones de un solo ejemplar, ya que se crea una tendencia poco útil a «curar por curar, o colectar por colectar» (21).

De acuerdo con los resultados del conjunto de los índices de biodiversidad, se hace necesario una revisión de objetivos concretos para las colecciones biológicas. Fernández (21), afirma que los problemas 
que afrontan los museos actualmente es debido a la falta de curatoría del material depositado en los museos, y que a su vez estos ejemplares no están asociados a catálogos ni a publicaciones. Es por eso, que los museos deben cumplir diferentes principios, dos de ellos de suma importancia: 1. que ocupen el menor espacio posible, pero a la vez conserven y muestren las propiedades consideradas de valor para su clasificación; 2. que en su recolección y estudio sean aplicadas las mismas técnicas, procedimientos, equipos y materiales, de modo que la comparación de atributos entre los distintos organismos sea estandarizada y no se oculten o compliquen las comparaciones, como resultado de no cuidar este «principio de homogeneidad»(26). Una colección ideal según Mcginley (27), debe basarse en la preservación y conservación de las colecciones por medio de la categorización en diez niveles, definida por la Política de Manejo de Colecciones, Departamento de Entomologia, USNM, Mayo 18 de 1992, de manera que permita clasificar todos los especimenes de acuerdo al nivel de curatoría y a las características propias de cada colección. Sin embargo, cuando un grupo biológico como el de Escleractinios del Caribe colombiano es tan reducido y se ha implementado recientemente su base de datos, la categorización se dará siempre en niveles altos, optima curatoría, ya que este grupo no demanda tantos ejemplares como si es el de artrópodos (21). También, se debe a que la colección de corales tiene personas expertas en taxonomía de este grupo, y periódicamente están alimentando la colección.

En general, los indicadores de biodiversidad utilizados para evaluar y establecer los patrones de información, ausencia- presencia de taxones e información documentada contenida en la base de datos de Escleractinios -MUJ, facilitará las decisiones de manejo, los esfuerzos de colecta, la inversión económica, y los recursos humanos necesarios para que el museo defina sus prioridades y logre metas concretas (6). Es de anotar, que la toma de decisiones, entre ellas, las relacionadas con la asignación de presupuesto, metas, autoevaluación y conservación para los museos depende de los elementos de juicio que parcialmente aportan herramientas concernientes con biodiversidad (28). Para ello, los indicadores deben utilizarse de manera conjunta, ya que esto permitirá conclusiones mas robustas acerca del estado general de las colecciones y de la biodiversidad que posee (6).

Por último, los indicadores mencionados son un camino para obtener información sobre la situación y estado de la biodiversidad en un país, ya que evidencian que hay frente a lo reportado, donde está, y tendencias espacio temporales de distribución de organismos (19). Así mismo, los indicadores de biodiversidad son herramientas de diagnóstico de las colecciones, al fundamentarse en los datos recopilados de inventarios taxonómicos. La ventaja de la aplicación de estos indicadores es que permiten abordar las colecciones desde diferentes perspectivas; cantidad, calidad de información, representatividad taxonómica y en consecuencia, tomar decisiones adecuadas para avanzar en la elaboración del inventario nacional de biodiversidad (4). Este hecho es soportado inicialmente con la prueba piloto de los indicadores aplicados a familias de las colecciones del IAvH: Scelionidae, Platygastridae, Proctotrupideae, Chalcididae, Pimplinae, Chrysidoidea, colección de Mariposas SchmidtMumm, Cerambycidae y Curculionidae, ya que los resultados sustentaron la propuesta metodológica y conceptual de los indicadores de conocimiento (17). Se concluye que los indicadores de biodiversidad basados en la información de las colecciones biológicas, deben aplicarse cuando existan objetivos claros, basados en las necesidades y prioridades institucionales y dependiendo de la cantidad y calidad de la información existente, siempre dentro del marco de referencia que es la biodiversidad. La veracidad de los indicadores de conocimiento sobre biodiversidad, como lo afirma Hernández (30) se refleja, dados los resultados del trabajo, en el interés de dar a conocer la información de las colecciones biológicas para cualquier usuario que 
desee obtener información sobre los atributos de organismos registrados, en el inventario taxonómico, en sus patrones de distribución espacial y temporal; en las regiones de colecta y época, así como en la facilitación de apoyo entre las colecciones biológicas teniendo en cuenta su complementariedad con otras y en la divulgación del conocimiento adquirido y compilado (30).

Es preciso anotar, que la colección de corales del MUJ, en términos generales presentó valores altos para la mayoría de los indicadores, debido a que la colecta de ejemplares se realizó teniendo en cuenta la recopilación de información de manera homogénea para todos los especimenes, y se tuvo sumo cuidado con la elaboración de las bases de datos, criterios y estándares antes mencionados; no obstante, es necesario aplicar conjuntamente con los indicadores de conocimiento los índices de salud propuestos para las prioridades sugeridas por Mcginley (21), de manera tal que en un futuro el material colectado no referenciado, no se pierda en el tiempo ni espacio, sino que pueda clasificarse de acuerdo a su nivel de estado (15), y de esta manera la inversión económica y humana por parte del museo permita asegurar el patrimonio biológico de la nación (23).

\section{Agradecimientos}

Este trabajo fue realizado dentro del proyecto "Organización y sistematización de las colecciones algales y de invertebrados no artrópodos de las colecciones biológicas del Museo Javeriano“ financiado por la Vicerrectoría de la Pontificia Universidad Javeriana (PUJ) Código 1583/ 2004.

\section{Referencias}

1. Winograd M, Cancino S. Hojas metodológicas del Sistema de Indicadores de Planificación y Seguimiento Ambiental (SIPSA) Proyecto CIAT-PNUMA: indicadores ambientales y de sustentabilidad. Unidad Política Ambiental DNP. Centro internacional de agricultura tropical (CIAT) III versión, Julio 2001. 28 p.

2. Ortiz N, et al. Sistema de indicadores de seguimiento de la Política de Biodiversidad en Colombia: Aspectos conceptuales y metodológicos. Instituto de Investigación de Recursos Biológicos Alexander von Humboldt. Serie: Indicadores de seguimiento y evaluación de la Política de Biodiversidad. 2004. 57 p.

3. Hawksworth D. Biodiversity: measurement and estimation.
Chapman and Hall: London. 1995. 140 p.

4. Instituto de Investigaciones y Recursos Biológicos Alexander von Humboldt. 2002. en: http://araneus.humboldt.org.co/ chmcolombia/servicios/jsp/foro_indicadores/ otros_inidcadores_conocimiento.htm

5. Meyer J. El juego de los indicadores. ¿Para qué y a quién sirve un observatorio de ciencia y tecnología en Colombia? Instituto francés de investigación científica para el desarrollo de la cooperación 1995. ORSTOM.

6. Vivas A. Evaluación de la información de colecciones biológicas sobre los géneros Elaeagia y Faramea (Rubiaceae) en la región andina colombiana. Sistema de Información sobre Biodiversidad - Instituto Alexander von Humboldt. Villa de Leyva, Colombia. 2003: 27 p. Versión 2.0 en:

URL:http://www.humboldt.org.co/sib/pdfs/indicadores/ Elaeagia\%20-\%20Faramea.pdf.

7. Instituto de Investigaciones y Recursos Biológicos Alexander von Humboldt (IAVH). 2002 en: http:// araneus.humboldt.org.co/chmcolombia/servicios/jsp/ foro indicadores/otros inidcadores conocimiento.htm

8. Agencia Europea para el Medio Ambiente (AEMA). Europe's environment - the 2nd assessment. Luxemburgo: Office for Publications of the European Communities. Elsevier Science. 1998.

9. Reyes J. Lista de los Corales (Cnidaria: Anthozoa: Scleractinia) de Colombia. Biota Colombiana 2000; 1 (2): 164 - 176.

10. Rivera H, Suárez A. Estándar para la documentación de metadatos de conjuntos de datos relacionados con biodiversidad. Instituto de Investigación de Recursos Biológicos Alexander von Humboldt, Bogotá D.C. Colombia 2002: 56 p.

11. Hyman G. Análisis espacial y sistemas de información geográfica para establecer prioridades regionales en la investigación y desarrollo agropecuario. Paper presented at Reunión Foro de las Américas para la Investigación y Desarrollo Tecnológico Agropecuario (FORAGRO) Sobre Prioridades Regionales de Investigación Centro Internacional de Mejoramiento de Maíz y Trigo (CIMMYT), mayo 2000. México.

12. Barnes R, Ruppert E. Zoología de los Invertebrados. Sexta edición Editorial McGraw-Hill interamericana: Mexico 1996: 1114 p.

13. Renjifo L, et al. Estrategia nacional para la conservación de las aves de Colombia. IAVH. Bogotá D.C. Colombia; Versión 2.12000.

14. Delgado, M.J. "Evaluación del Potencial de Integración y Análisis de Registros de Aves en Colombia". Trabajo de Grado - Carrera de Biología - Facultad de Ciencias - Pontificia Universidad Javeriana. Bogotá D.C. 2001. 52 p.

15. IAvH, Conservación Internacional- Colombia \& Departamento nacional de planeación- Misión Social. Sistema de indicadores de seguimiento y evaluación de la política en Biodiversidad en los Andes colombianos -Informe FinalBogotá D.C. Colombia 2000.

16. Campos D. Avispas parasíticas de la familia Braconidae (Hymenoptera) en Colombia: Diversidad genérica y distribución geográfica. Tesis de Pregrado Biología Universidad Nacional de Colombia. Bogotá D.C. 2001. 70 p. En Vivas A. Zalamea, M. IAvH. Sistema de Información sobre Biodiversidad SIB. Propuesta para la implementación de un Sistema de Indicadores del estado de Conocimiento sobre Biodiversidad para Colombia Elaborada en el marco del Proyecto específico Construcción de una Línea de Base sobre el Estado de la Biodiversidad en Colombia- Versión 2.1. Villa de Leiva Colombia 200337 p.

17. Vivas A, Zalamea M. Instituto de Investigación de Recursos Biológicos "Alexander von Humboldt". Sistema de Información sobre Biodiversidad SIB. Propuesta para la implementación de un Sistema de Indicadores del estado de Conocimiento sobre biodiversidad para Colombia - Elaborada en el marco del Proyecto específico Construcción de una Línea de Base sobre el Estado de la Biodiversidad en Colombia- Versión 2.1 Villa de Leiva Colombia 2003. 37 p.

18. Soberón J. El compromiso de ser un país megadiverso: El Sistema Nacional de Información sobre Biodiversidad. Desarrollo Sustentable febrero 2000: p. 28-31.

19. Rudas G. Reflexiones sobre el Diseño un Sistema de Indicadores 
de Seguimiento de la Política de Biodiversidad. Documento de Trabajo, no publicado, IAvH. Programa de Investigación en Política y Legislación. Proyecto Sistema de Indicadores de la Biodiversidad. Bogotá D.C. Colombia 1999. 179 p.

20. LlorenteJ, González E, Papavero N. Biodiversidad: Taxonomía y Biogeografía de Artrópodos de México: Hacia una Síntesis de su Conocimiento. Universidad Nacional Autónoma de México; 2000. 676 p.

21. Fernández F. La Gestión en la Administración de Museos de Historia Natural. IAvH. Memorias XXVI. Congreso Sociedad Colombiana de Entomologia 2000: 253-264.

22. Villareal $\mathrm{H}$, et al. Manual de métodos para el desarrollo de inventarios de biodiversidad. IAvH. 2004 p.

23. Williams P, Gaston K. Measuring more of biodiversity: Can higher-taxon richness predict wholesale species richness? Biological Conservation. 1994; 67:211-217.

24. Colwell R, Coddington J. Estimating terrestrial biodiversity throught extrapolation. 101-118. In Hawksworth, D: L. (ed.): the quantification and estimation of organismal biodiversity. Special volume, Phil. Trans. R, Soc. London. 1996; 140 p.

25. Hijmans R, et al. Assessing the geographic representativeness of genebank collections: the case of Bolivian wild potatoes. Conservation Biology. 2000;14(6): 1755-1765.
26. Llorente J, Luna, compiladores. Bases de datos en taxonomía y colecciones científicas, Taxonomía biológica. Ediciones Científicas Universitarias UNAM, Fondo de Cultura Económica. Mexico: 1994. $148 \mathrm{p}$.

27. Mcginley R. Where's the Management in Collections Manegement?. In Congress Book, vol 3, Current Issues, initiatives, and future directions for the Preservation and Conservation of Natural History colletions, Madrid;1993. 338 p.

28. Soberón J, Llorente J, Oñate L. The use of specimen-label databases for conservation purposes: An example using Mexican Papilionid and Pierid butterflies. Biodiversity and Conservation. 2000; 9: 1441-1466.

29. Chávez M, Arango N. Informe nacional sobre el estado de la biodiversidad 1997 Colombia. IAvH, Ministerio del Medio Ambiente PNUMA. Bogotá D.C. Colombia. 1998. Vol 3.

30. IAvH. Biodiversidad y propiedad intelectual: la propiedad intelectual en la Organización Mundial del Comercio y su relación con el Convenio sobre Diversidad Biológica. Ana María Hernández. Bogotá D.C. Colombia 1999. 58 p. 